\title{
Is Rotational Thromboelastometry the Answer for Rapid Prediction of Coagulopathy on Extracorporeal Membrane Oxygenation?
}

\author{
Ameya Karanjkar ${ }^{1}$ Vandana Bhardwaj ${ }^{1}$ Poonam Malhotra Kapoor ${ }^{1}$ \\ ${ }^{1}$ Department of Cardiac Anaesthesia, Cardio Thoracic Centre, All \\ India Institute of Medical Sciences (AIIMS), New Delhi

\begin{abstract}
Address for correspondence Poonam Malhotra Kapoor, MD, DNB MNAMS, FIACTA (Hony), FTEE (Hony), FISCU (Hony), Department of Cardiac Anaesthesia, Cardio Thoracic Centre, All India Institute of Medical Sciences (AlIMS), New Delhi (e-mail: drpoonamaiims@gmail.com).
\end{abstract}
Abstract
Keywords
- coagulopathy
- extracorporeal mem- brane oxygenation
- rapid prediction
- rotational
thromboelastometry

Extracorporeal membrane oxygenation (ECMO) induces a variety of changes in hemostatic system due to various reasons such as exposure to the extracorporeal circuit, activation of the coagulation and complement pathways, consumption of platelets, activation of various mediators of inflammation, and imbalance between clot formation and resorption. Giving cryoprecipitate and platelet concentrates is more important than adding whole blood in these patients. EXTEM CT and FIBTEM A5 give results within a few minutes and shortened turnaround time brings imported results.

\section{Introduction}

Extracorporeal membrane oxygenation (ECMO) induces a variety of changes in hemostatic system due to various reasons such as exposure to the extracorporeal circuit, activation of the coagulation and complement pathways, consumption of platelets, activation of various mediators of inflammation, and imbalance between clot formation and resorption.

\section{Coagulopathy on Extracorporeal Membrane Oxygenation}

When the patient's blood first meets the foreign surface of the extracorporeal circuit, a variety of coagulative and inflammatory cascades are activated. Levels of proinflammatory cytokines such as tumor necrosis factor- $\alpha$ (TNF- $\alpha$, interleukin 6 , interleukin 8 , and bradykinin rise rapidly which in association with activation of the complement system results in activation of leukocytes. ${ }^{2}$

The complement system gets activated as soon as there is contact with the foreign ECMO circuit. Complement system, when activated, progresses to produce membrane attack complex (MAC) that further propagates the inflammatory response. $^{3}$
Extracorporeal support is associated with a disseminated intravascular coagulation (DIC)-like consumptive coagulopathy due to tissue injury and contact activation. It is characterized by widespread activation of coagulation resulting in fibrin deposition and microthrombi formation. The platelet and coagulation factor consumption associated with DIC can lead to severe bleeding and thrombosis both. It also causes adenosine diphosphate (ADP), arachidonic acid (ARA), and tartrate-resistant acid phosphatase (TRAP) platelet receptor dysfunction. ${ }^{4}$

Exposure to heparin is also an important cause of bleeding in ECMO patients. It may lead to heparin-induced thrombocytopenia and excessive anticoagulation. Both these factors contribute to the morbidity and mortality on ECMO patients. ${ }^{5}$

\section{Monitoring the Coagulopathy on Extracorporeal Membrane Oxygenation}

A blood clot is typically composed of red blood cells making up greater than $90 \%$ of blood clot volume. Clot strength is derived from the interaction of the fibrin network and platelets. Fibrin-based clot strength is dependent mainly on factor XIII and fibrinogen, whereas platelets contribute to overall clot strength by binding and tightening fibrin fibers. ${ }^{6}$

Copyright $\odot 2017$ Official Publication of The Simulation Society (TSS), accredited by International Society of Cardiovascular Ultrasound (ISCU)
License terms

(ㅇ)(1) $\ominus \circledast$
$10.1055 / \mathrm{s}-0038-1624063$ ISSN 2457-0206 
Classically, standard tests such as prothrombin time (PT), activated partial thromboplastin time (aPTT), international normalized ratio (INR), platelet count, and serum fibrinogen level are used to diagnose and treat the coagulopathy on ECMO. However, these tests have more turnover time. Recently, evaluation using rotational thromboelastogram (ROTEM) has gained popularity due to bedside availability and short turnover time (10-15 minutes). Parameters such as maximal clot firmness (MCF), amplitude at 5 minutes (A5) and 10 minutes (A10) accurately predict the function of coagulation system. A5 can provide an accurate estimate of coagulation function in 5 minutes. Platelet aggregometry using ROTEM platelet gives important information regarding qualitative function of platelets. ${ }^{7,8}$

The authors would like to discuss case of a 30-day-old, 3.2-kg infant diagnosed with D-transposition of great arteries. The patient was posted for arterial switch operation with integrated ECMO due to severe pulmonary hypertension. General anesthesia was induced, and cardiopulmonary bypass ( $\mathrm{CPB}$ ) was conducted with standard institutional protocols. After completion of procedure, the patient was put on venoarterial ECMO using poly methyl pentane circuit and Sarns centrifugal pump (Terumo Cardiovascular Systems Corp.).

After 40 hours of run on ECMO, the patient developed increased chest drain output. ROTEM analysis revealed deranged intrinsic and extrinsic pathways, and fibrinogen function (-Fig. 1).

To treat the coagulopathy, first fresh frozen plasma, then platelets, and finally 8 units of cryoprecipitate were transfused in massive doses. After repeated transfusion of the blood products, chest drain output decreased, but bleeding did not stop completely suggesting an undiagnosed cause.

Though EXTEM and FIBTEM provide information regarding clot strength and fibrinogen levels, it cannot assess the platelet dysfunction. However, qualitative and quantitative platelet changes occur when a neonate receives ECMO. Platelet aggregometry using ROTEM platelet can be useful in such cases

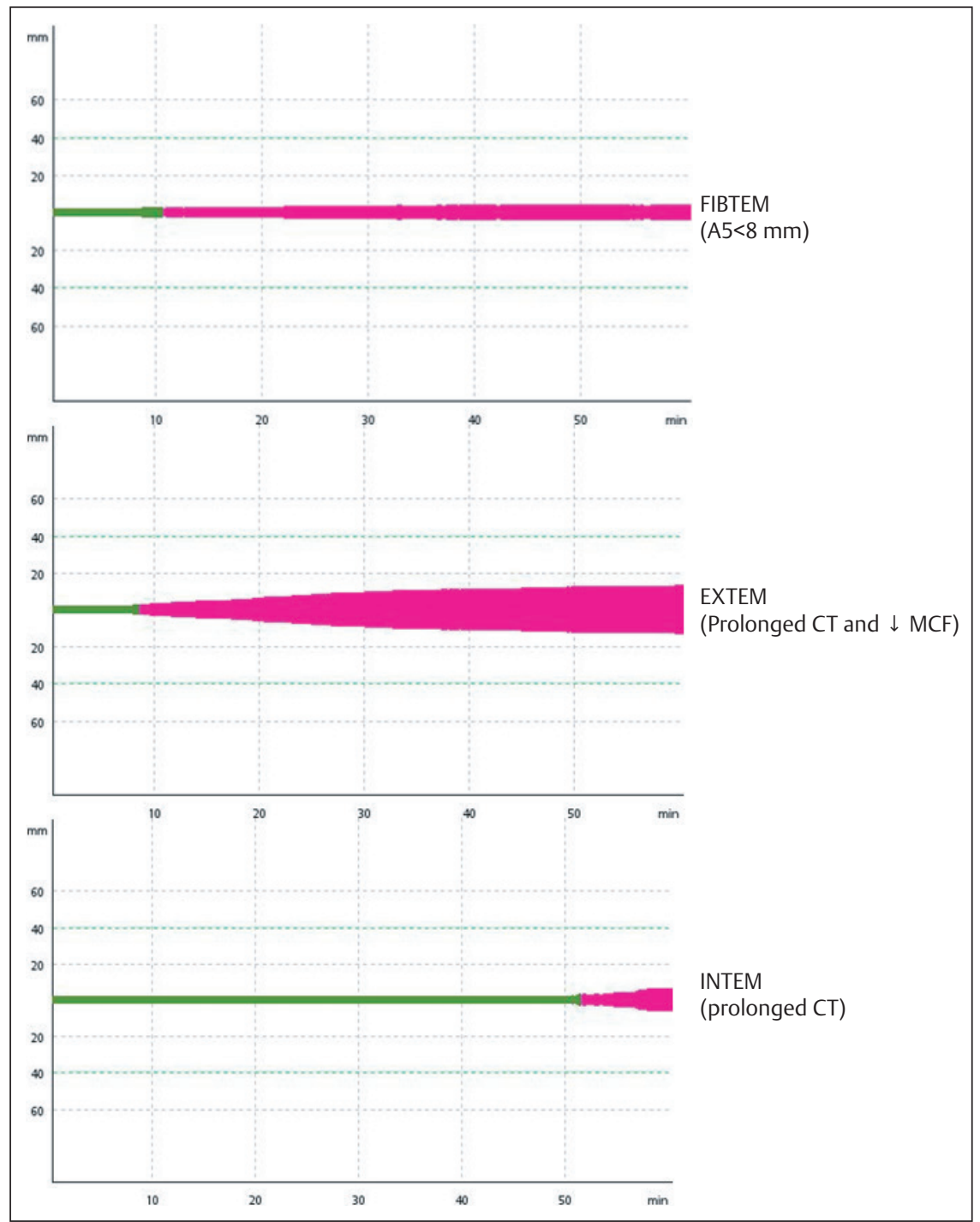

Fig. 1 Analysis using ROTEM. Upper figure shows deranged fibrinogen function, middle shows deranged extrinsic pathway, and the lower shows deranged intrinsic pathway. 
for further investigating the cause for bleeding, which may reveal the platelet dysfunction at ADP, TRAP, or ARA receptors.

\section{Conclusion}

ROTEM is an accurate and rapid tool for analysis of coagulation pathways. There is both qualitative and quantitative platelet dysfunction on ECMO that cannot be assessed by standard tests. Platelet aggregometry can come up as a valuable tool to assess the platelet dysfunction.

\section{Conflict of Interest}

None.

\section{References}

1 Esper SA, Levy JH, Waters $\mathrm{JH}$, Welsby IJ. Extracorporeal membrane oxygenation in the adult: a review of anticoagulation monitoring and transfusion. Anesth Analg 2014; 118(4):731-743

2 Millar JE, Fanning JP, McDonald CI, McAuley DF, Fraser JF. The inflammatory response to extracorporeal membrane oxygenation (ECMO): a review of the pathophysiology. Crit Care 2016; 20(1):387
3 Graulich J, Walzog B, Marcinkowski M, et al. Leukocyte and endothelial activation in a laboratory model of extracorporeal membrane oxygenation (ECMO). Pediatr Res 2000; 48(5):679-684

4 Plötz FB, van Oeveren W, Bartlett RH, Wildevuur CR. Blood activation during neonatal extracorporeal life support. J Thorac Cardiovasc Surg 1993;105(5):823-832

5 Gray BW, Haft JW, Hirsch JC, Annich GM, Hirschl RB, Bartlett RH. Extracorporeal life support: experience with 2,000 patients. ASAIO J 2015;61(1):2-7

6 Solomon C, Ranucci M, Hochleitner G, Schöchl H, Schlimp CJ. Assessing the methodology for calculating platelet contribution to clot strength (platelet component) in thromboelastometry and thromboelastography. Anesth Analg 2015; 121(4):868-878

7 Olde Engberink RH, Kuiper GJ, Wetzels RJ, et al. Rapid and correct prediction of thrombocytopenia and hypofibrinogenemia with rotational thromboelastometry in cardiac surgery. J Cardiothorac Vasc Anesth 2014;28(2):210-216

8 Bhardwaj V, Malhotra P, Hasija S, Chowdury UK, Pangasa $\mathrm{N}$. Coagulopathies in cyanotic cardiac patients: an analysis with three point-of-care testing devices (thromboelastography, rotational thromboelastometry, and Sonoclot analyzer). Ann Card Anaesth 2017;20(2):212-218 